\title{
Thiamine Prevents Obesity and Obesity-Associated Metabolic Disorders in OLETF Rats
}

\author{
Takao TANAKA ${ }^{1,2}$, Tatsuji KonO ${ }^{2}$, Fumio TerasaKI ${ }^{2}$, Kota YASUI ${ }^{1}$, Akiko SOYAMA ${ }^{2}$, \\ Kaoru OTSUKA ${ }^{2}$, Shuichi FujtTA ${ }^{2}$, Kazuhiko YAmAnE ${ }^{1}$, Miki MANABE ${ }^{1}$, Kazuya Usui ${ }^{1}$ \\ and Yuka KOHDA ${ }^{1}$ \\ ${ }^{1}$ Laboratory of Pharmacotherapy, Osaka University of Pharmaceutical Sciences, Nasahara 4-20-1, \\ Takatsuki, Osaka 569-1094, Japan \\ ${ }^{2}$ Third Division, Department of Internal Medicine, Osaka Medical College, Daigaku-cho 2-7, \\ Takatsuki, Osaka 569-8686, Japan
}

(Received April 28, 2010)

\begin{abstract}
Summary We previously found that thiamine mitigates metabolic disorders in spontaneously hypertensive rats, harboring defects in glucose and fatty acid metabolism. Mutation of thiamine transporter gene SLC19A2 is linked to type 2 diabetes mellitus. The current study extends our hypothesis that thiamine intervention may impact metabolic abnormalities in Otsuka Long-Evans Tokushima Fatty (OLETF) rats, exhibiting obesity and metabolic disorders similar to human metabolic syndrome. Male OLETF rats (4 wk old) were given free access to water containing either $0.2 \%$ or $0 \%$ of thiamine for 21 and $51 \mathrm{wk}$. At the end of treatment, blood parameters and cardiac functions were analyzed. After sacrifice, organs weights, histological findings, and hepatic pyruvate dehydrogenase (PDH) activity in the liver were evaluated. Thiamine intervention averted obesity and prevented metabolic disorders in OLETF rats which accompanied mitigation of reduced lipid oxidation and increased hepatic PDH activity. Histological evaluation revealed that thiamine alleviated adipocyte hypertrophy, steatosis in the liver, heart, and skeletal muscle, sinusoidal fibrosis with formation of basement membranes (called pseudocapillarization) which accompanied significantly reduced expression of laminin $\beta 1$ and nidogen-1 mRNA, interstitial fibrosis in the heart and kidney, fatty degeneration in the pancreas, thickening of the basement membrane of the vasculature, and glomerulopathy and mononuclear cell infiltration in the kidney. Cardiac and renal functions were preserved in thiamine treatment. Thiamine has a potential to prevent obesity and metabolic disorders in OLETF rats.
\end{abstract}

Key Words thiamine, obesity, metabolic syndrome, OLETF rats

Obesity is a condition with major clinical relevance to metabolic disorders, such as insulin resistance, type 2 diabetes, steatosis, hypertension and dyslipidemia. It also leads to proinflammatory and prothrombotic states that potentiate atherosclerosis (1). The incidence of obesity is expected to significantly increase due to changing eating habits (increased overall caloric intake from lipids and simple sugars) and decreasing physical activity. In 2005, the World Health Organization estimated that more than 400 million adults worldwide were obese, and this incidence continues to increase at an alarming rate (2). Because of the increasing number of dire health problems in many countries, it is of utmost importance to develop novel preventive strategies for obesity. Current strategies include diet and exercise; nevertheless, an effective pharmaceutical arm remains to be provided.

Obesity frequently leads to type 2 diabetes mellitus. Even though substantial clinical and experimental evidence suggest beneficial impacts of thiamine supplementation on diabetic complications, such as diabetic retinopathy, diabetic nephropathy, diabetic neuropathy

E-mail: in3024@poh.osaka-med.ac.jp and diabetic cardiomyopathy (3-10), no report has investigated the prevention of the onset or development of type 2 diabetes mellitus due to thiamine intervention. Nonetheless, thiamine is possibly connected to the commencement of diabetes mellitus and metabolic disorders.

Thiamine plays crucial roles in cellular metabolism, and impaired thiamine uptake results in a variety of disorders. Thiamine-responsive megaloblastic anaemia (TRMA), also known as Rogers syndrome, is an earlyonset, autosomal recessive disorder defined by the occurrence of megaloblastic anaemia, diabetes mellitus and sensorineural deafness, which responds to thiamine treatment in varying degrees. TRMA is a rare genetic disorder in which diabetes, other than type 1 diabetes mellitus, is secondary to a single gene disorder, a thiamine transporter defect (11-14). Type 2 diabetes mellitus has been linked to the chromosome 1q23.223.3, site of the SLC19A2 gene $(15,16)$. Furthermore, although a relationship of cause and consequent effect has not been elucidated, studies indicate that mild thiamine deficiency is prevalent in diabetes $(17,18)$.

Many of the defining features of human metabolic syndrome, in which hypertension is associated with 
insulin resistance, dyslipidemia and abdominal obesity, are manifest in the spontaneously hypertensive rat (SHR) (19). The quantitative trait locus for glucose and fatty acid metabolism in SHR was identified as a defective CD36 gene (also known as FAT because it encodes fatty acid translocase) and CD36 deficiency has been proposed to underlie insulin resistance, defective fatty acid metabolism and hypertriglyceridemia in SHR (20). CD36 deficiency results in defective fatty acid uptake, thus in turn giving rise to reliance on glucose as an energy source. Based on the assumption that excess glucose influx causes relative thiamine deficiency, we provided thiamine-containing water to CD36-defective SHR. Thiamine intervention attenuated hypertension, body weight gain and metabolic abnormalities in CD36defective SHR (21).

Taking these findings into consideration, it seemed rational to hypothesize that thiamine deficiency could be an underlying mechanism in type 2 diabetes mellitus. This study expands our hypothesis that excess carbohydrate intake could be accompanied by a thiaminedeficient condition. In this study, we chose Otsuka Long-Evans Tokushima Fatty (OLETF) rats as models for studying human obesity and metabolic disorders. OLETF rats lack functional cholecystokinin-A receptors, resulting in polyphagia, and have been extensively studied as models for obesity and metabolic disorders similar to human metabolic syndrome. We report here that thiamine intervention has the potential to prevent obesity and multiple features of obesity-associated metabolic disorders.

\section{MATERIALS AND METHODS}

Animals and protocols. The animal protocols were approved by the Institutional Animal Care and Use Committee of Osaka University of Pharmaceutical Sciences. OLETF male rats at $4 \mathrm{wk}$ of age were kindly supplied by the Tokushima Research Institute, Otsuka Pharmaceutical (Tokushima, Japan). OLETF rats were randomly divided into untreated control and thiaminetreated groups of 16 rats each, in which 8 rats were sacrificed at $25 \mathrm{wk}$ of age and the remaining 8 rats were sacrificed at $55 \mathrm{wk}$ of age. The latter group was provided $2 \mathrm{~g}$ thiamine/ $\mathrm{L}$ in their drinking water. Rats were housed in standard cages (2 rats per cage) with free access to water and standard rodent chow (NMF, Oriental Yeast Co., Ltd., Tokyo, Japan). The thiamine dose chosen was consistent with the dose used in a previous study done on SHR (21) and was comparable with that used for the treatment of diabetic complications $(3,6)$. Cages were kept in temperature-controlled animal quarters $\left(21^{\circ} \mathrm{C}\right)$ with a 0600-1800 light : 1800-0600 dark cycle, which was maintained throughout the experimental period. Body weights were measured between 0800 and $1000 \mathrm{~h}$. Body weight as well as food and water intake were measured weekly throughout the experimental period.

One week before sacrificing the rats, systolic blood pressure and heart rates were measured using a noninvasive tail cuff and pulse transducer system (BP-98A;
Softron, Tokyo, Japan). Urinary albumin was quantified in 24-h urine collections from rats kept in individual metabolic cages using the enzyme linked immunosorbent assay (ELISA; Nephrat, Exocell, PA, USA). The concentration of urinary creatinine was determined by alkaline picrate using a test kit (Wako Pure Chemical Industries, Ltd., Osaka, Japan). Urinary albumin levels were expressed as albumin-to-creatinine ratios.

Blood and tissue sampling. Control and thiaminetreated rats were sacrificed at 25 and $55 \mathrm{wk}$ of age (thiamine treatments for 21 and $51 \mathrm{wk} ; n=8$ per group). After $16 \mathrm{~h}$ of fasting, rats were anaesthetized with pentobarbital $(50 \mathrm{mg} / \mathrm{kg})$ and indwelling catheters were inserted into right carotid arteries. Blood samples were collected from the arteries into EDTA tubes. Plasma was separated from whole blood within $30 \mathrm{~min}$ by centrifugation in a refrigerated bench-top centrifuge, and aliquots of plasma were stored at $-80^{\circ} \mathrm{C}$ until further analysis.

From exsanguinated animals, samples of heart, skeletal muscle (gastrocnemius muscle), liver, kidneys, pancreas, epididymal fat pads and retroperitoneal fat pads were removed, rinsed in ice-cold saline, briefly blotted with paper and weighed. Organs were either snap-frozen in liquid nitrogen or placed in an RNAlater (Ambion, Austin, TX, USA; $-80^{\circ} \mathrm{C}$ ) or $10 \%$ formalin, until further analysis.

Echocardiography. The animals, at $55 \mathrm{wk}$ of age, were lightly anaesthetized by intraperitoneal administration of $10 \mathrm{mg} / \mathrm{kg}$ xylazine (Selactar, Bayer Yakuhin, Tokyo, Japan) and $50 \mathrm{mg} / \mathrm{kg}$ ketamine (Ketaral, Daiichi Sankyo Co., Ltd., Tokyo, Japan). Two-dimensional guided M-mode echocardiography was performed using an instrument equipped with a multi-frequency transducer and Doppler tissue imaging (DTI) program (Vivid 5, GE Medical Systems, Milwaukee, WI, USA). The heart was imaged in a two-dimensional mode in a parasternal short-axis view with a depth setting of $2 \mathrm{~cm}$. From this view, an M-mode cursor was positioned perpendicular to the interventricular septum and posterior wall of the LV at the level of the papillary muscles. An M-mode image was obtained at a sweep speed of $100 \mathrm{~mm} / \mathrm{s}$. Left ventricular (LV) end diastolic diameter (Dd), LV end systolic diameter (Ds), diastolic wall thickness of the interventricular septum (IVSd) and posterior wall thickness of LV (PWd) were obtained from M-mode tracings of measurements averaged from 3 separate cardiac cycles. All measurements were performed from leading edge to leading edge according to the American Society of Echocardiography guidelines. LV systolic function was determined by estimating the LV ejection fraction (LVEF) and LV fractional shortening (FS). FS was derived from the equation: $\mathrm{FS}=((\mathrm{Dd}-\mathrm{Ds}) / \mathrm{Dd}) \times$ 100. LVEF was calculated using the multiple diameter method. For evaluation of diastolic function, mitral inflow velocities were recorded using pulsed-wave Doppler at the tips of the mitral valve leaflets (early diastolic flow (E) and atrial contraction flow (A) waves, $\mathrm{cm} / \mathrm{s}$ ). A deceleration time of $\mathrm{E}(\mathrm{Edec})$ was measured as the period between the peak $\mathrm{E}$ velocity and the point where the 
slope met the baseline. The early diastolic velocity recorded at the lateral corner of the mitral annulus (Ea), a preload-independent index of $\mathrm{LV}$ relaxation, was also obtained with DTI.

Metabolic parameters. Plasma glucose, total cholesterol (T-Cho), triglyceride (TG), ketone bodies and nonesterified fatty acid (NEFA) concentrations were measured on an automated JCA-BM8001 analyzer (JEOL Ltd., Tokyo, Japan) using various commercial kits. Glucose: the SHINO-TEST (Tokyo, Japan); T-Cho: an L-type CHO H kit (Wako Pure Chemical Industries, Ltd.); TG: an L-type TG H kit (Wako Pure Chemical Industries, Ltd.); ketone bodies: Total ketone body KAINOS (KAINOS Laboratories Inc, Tokyo, Japan); NEFAs: the NEFA C-test kit (Wako Pure Chemical Industries, Ltd.). Glycated haemoglobin (HbA1c) levels were measured using a latex agglutination method (RAPIDIA Auto HbA1c-L; Fujirebio Inc., Tokyo, Japan). Plasma insulin concentrations were assayed using the Rat/Mouse Insulin ELISA kit (LINCO Research, St. Charles, MO, USA).

Determination of hepatic TG contents and pyruvate dehydrogenase (PDH) activity. To determine the accumulation of TGs within the livers, TGs were extracted from approximately $50 \mathrm{mg}$ of liver in chloroform-methanol (22) and quantified using the L-type TG H kit, which was calibrated against a triolein TG standard (23). PDH activity was assayed using the radioenzymatic method of Sterk et al. (24). All measurements were made at $37^{\circ} \mathrm{C}$. For determination of the active PDH fraction the assay mixture contained, in $100 \mu \mathrm{L}$ final volume: $50 \mathrm{~mm}$ Hepes (pH 7.2), $2 \mathrm{mM} \mathrm{MgCl}_{2}, 3.35 \mathrm{mM} \mathrm{NAD}$, $0.4 \mathrm{mM}$ TPP, $0.45 \mathrm{mM}$ CoA, $10 \mathrm{mM}$ L-carnitine, 0.08 mM EGTA, $2.5 \mathrm{~mm} \mathrm{NaF,} 0.8$ unit carnitine acetyltransferase (dialyzed overnight against $50 \mathrm{~mm}$ potassium phosphate buffer, $\mathrm{pH} 7.4$ ), and $5 \mu \mathrm{L}$ of a $10 \%$ rat liver homogenate solubilized with octylglucoside. Active PDH activity was expressed as nanomoles of acetylcarnitine formed per minute and per milligram of protein.

Histological evaluations. Removed organs (heart, gastrocnemius muscle, liver, kidneys, pancreas and retroperitoneal and epididymal fat pads) were immediately fixed in $10 \%$ phosphate-buffered formalin solution and then embedded in paraffin. Paraffin-embedded sections ( $4 \mu \mathrm{m})$ were routinely stained with haematoxylin and eosin (H\&E) for overall morphology. Images were recorded using a light microscope (Nikon, Tokyo, Japan) and then stored electronically using microscopic imageanalyzing equipment (Nikon microscope Eclipse E800, Nikon). Collagen, neutral lipids, insulin and renal histopathology were analyzed using special stains as follows.

Fibrosis. Paraffin-embedded sections $(4 \mu \mathrm{m})$ were stained with Picric Acid Sirius Red for collagen analysis. Myocardial and renal interstitial collagen volume percentages in the LV midwall and whole kidney were quantitatively evaluated with a high-quality scanner (GT-X800, Seiko Epson Co., Tokyo, Japan) using Scion Image Beta 4.02 software (Scion Corporation, Frederick, MD, USA).

Neutral lipids. For examination of intracellular lipid accumulations in heart and skeletal muscles, the tis- sues were formalin-fixed and immersed in 30\% sucrose. These were then embedded in an optimal cutting temperature compound, frozen in methylbutane over liquid nitrogen and cut into $4-\mu \mathrm{m}$ sections. Slices were placed in $60 \%$ isopropyl alcohol solution for $1 \mathrm{~s}$ followed by incubation for $1 \mathrm{~h}$ in Oil Red O stain. Samples were then briefly (1-3 s) rinsed with 60\% isopropyl alcohol, washed for 3 min under running tap water and counterstained with haematoxylin.

Insulin. For evaluations of $\beta$-cells, sections of each pancreas were incubated with guinea pig anti-insulin polyclonal antibody (Biomeda, Foster, CA, USA), followed by signal amplification using a VECTASTAIN Elite ABC Kit (PK-6102; Vector Laboratories, Burlington, CA, USA). The reaction was developed by addition of AEC chromogen substrate (AEC Staining Kit; SigmaAldrich Co., St. Louis, MO, USA).

Renal histopathological analysis. Sections (4- $\mu \mathrm{m})$ from each kidney were cut and stained with periodic acid methenamine-silver stain (PAM) and periodic acidSchiff (PAS) reagents. Renal histopathology was assessed for glomerular hypertrophy (glomerulomegaly), segmental glomerulosclerosis and thickening of the glomerular basement membrane using H\&E, PAS and PAM, respectively as follows: Glomerulomegaly was evaluated based on the area of glomeruli observed on a PC monitor using digital measurement software. Based on PAS staining, extracellular matrix (ECM) involving more than $50 \%$ of the glomerular area was arbitrarily assessed as positive for segmental glomerulosclerosis. Robust staining with PAM was arbitrarily assessed as positive for thickening of the glomerular basement membrane. Glomerulomegaly, segmental glomerulosclerosis and thickening of basement membrane were evaluated for approximately 50 glomeruli per animal ( $n=4$, for both thiamine-treated and control groups).

Quantitative RT-PCR analysis. Quantitative RT-PCR analysis was performed using the LightCycler RealTime PCR System (Roche Molecular Biochemicals, Indianapolis, IN, USA) to detect the mRNA expression of nidogen- 1 and laminin $\beta 1$. The expression of $\beta$-actin mRNA was measured as the internal control. Assays were designed using the Roche Universal Probe Library (https://www.roche-applied-science.com/sis/rtpcr/upl/ index.jsp). For each reaction, the LightCycler Taqman Master Kit (Roche Molecular Biochemicals) was used according to the manufacturer's instructions. PCR cycling was conducted under the following conditions: preheating for 1 cycle at $95^{\circ} \mathrm{C}$ for $10 \mathrm{~min}$, amplification for 45 cycles at $95^{\circ} \mathrm{C}$ for $10 \mathrm{~s}$ and $60^{\circ} \mathrm{C}$ for $25 \mathrm{~s}$, and final cooling to $40^{\circ} \mathrm{C}$. mRNA levels were quantified and normalized against levels of $\beta$-actin. The averaged and normalized levels of mRNA in each control group were expressed as 1.0.

Statistics. Each outcome measure was examined in 4 to 8 animals. Results are reported as means \pm standard error. Statistical analyses used the Microsoft Excel Data Analysis ToolPak (Stacel2, OMS Publish Inc., Tokorozawa, Japan). Group comparisons were performed using a 2-tailed Student's $t$ test. Statistical dif- 
a

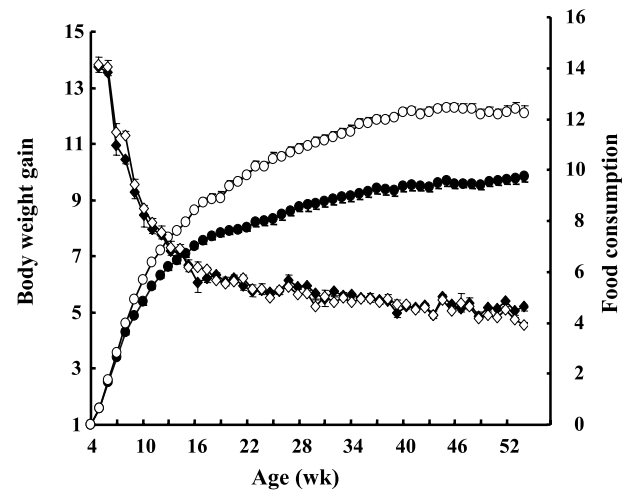

b

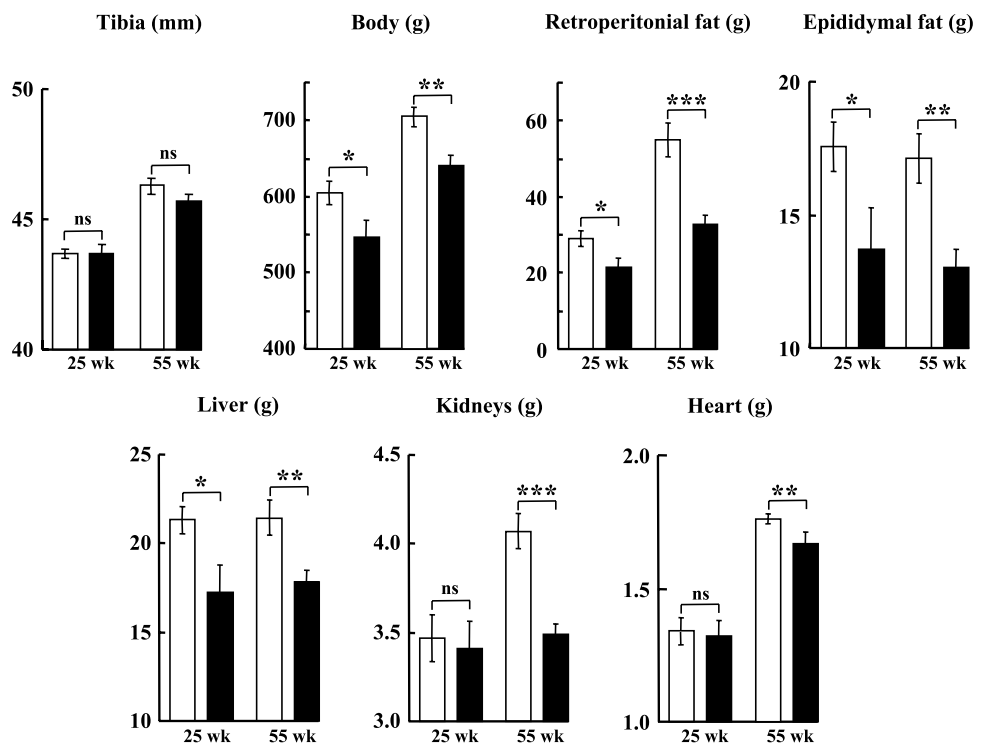

Fig. 1. Thiamine-associated decrease in body and organ weights in OLETF rats. a: Male OLETF rats (4 wk old) were provided with water (control group, open circle) or water containing $0.2 \%$ thiamine (thiamine group, closed circle). Body weight gain is expressed as magnitude of change relative to baseline body weight. Food consumption relative to body weight (control group, open diamond; thiamine group, closed diamond), calculated as food consumption (g) divided by body weight ( $\mathrm{g} / 100)$, was measured weekly, and all values are expressed as mean \pm standard error (SE) for $n=16$ rats until $21 \mathrm{wk}$ and $n=8$ rats until $51 \mathrm{wk}$ in each group. Body weight gain in the thiamine group was significantly less than that in the control group beginning $4 \mathrm{wk}$ after treatment $(p<0.02)$ and throughout the remaining experimental period $(p<0.001)$. b: Weights of organs harvested at 25 and $55 \mathrm{wk}$ are shown in bar graphs. Open columns indicate control OLETF rats. Closed columns indicate thiamine-treated OLETF rats. Bars indicate means \pm SE for $n=8 .{ }^{*} p<0.05 ;{ }^{* *} p<0.01$; *** $p<0.001$ by an unpaired, two-tailed Student's $t$ test.

Table 1. Body weight and metabolic parameters in LETO and OLETF rats.

\begin{tabular}{|c|c|c|c|c|}
\hline & $25 \mathrm{wk}$ & $30 \mathrm{wk}$ & $50 \mathrm{wk}$ & $55 \mathrm{wk}$ \\
\hline \multicolumn{5}{|l|}{ Body weight (g) } \\
\hline LETO-Breeder & & $528 \pm 22$ & $584 \pm 26$ & \\
\hline OLETF-Breeder & & $659 \pm 42$ & $682 \pm 68$ & \\
\hline OLETF-Control & $611.1 \pm 18.9$ & $649.3 \pm 19$ & $707.3 \pm 19$ & $704.8 \pm 28.3$ \\
\hline OLETF-Thiamine & $543.1 \pm 20.5$ & $582.3 \pm 17$ & $629.8 \pm 15$ & $641.1 \pm 18.1$ \\
\hline \multicolumn{5}{|l|}{ Glucose (mM) } \\
\hline LETO-Breeder & & $6.61 \pm 0.56$ & $7.22 \pm 0.50$ & \\
\hline OLETF-Breeder & & $8.99 \pm 1.22$ & $14.54 \pm 7.1$ & \\
\hline OLETF-Control & $11.34 \pm 0.51$ & & & $14.41 \pm 0.55$ \\
\hline OLETF-Thiamine & $8.95 \pm 0.43$ & & & $10.89 \pm 0.39$ \\
\hline \multicolumn{5}{|l|}{ Triglyceride (mM) } \\
\hline LETO-Breeder & & $1.01 \pm 0.20$ & $1.02 \pm 0.25$ & \\
\hline OLETF-Breeder & & $3.32 \pm 1.24$ & $3.37 \pm 0.52$ & \\
\hline OLETF-Control & $1.84 \pm 0.20$ & & & $3.15 \pm 0.60$ \\
\hline OLETF-Thiamine & $1.02 \pm 0.20$ & & & $1.56 \pm 0.28$ \\
\hline \multicolumn{5}{|l|}{ Total cholesterol (mM) } \\
\hline LETO-Breeder & & $2.46 \pm 0.31$ & $2.33 \pm 0.03$ & \\
\hline OLETF-Breeder & & $3.16 \pm 0.62$ & $4.50 \pm 0.65$ & \\
\hline OLETF-Control & $2.90 \pm 0.16$ & & & $4.58 \pm 0.17$ \\
\hline OLETF-Thiamine & $2.39 \pm 0.12$ & & & $3.05 \pm 0.12$ \\
\hline
\end{tabular}

LETO-Breeder, LETO rats bred at the Tokushima Research Institute, Otsuka Pharmaceutical (Tokushima, Japan); OLETFBreeder, OLETF rats bred at the Tokushima Research Institute, Otsuka Pharmaceutical; OLETF-Control, untreated OLETF rats in this study; OLETF-Thiamine, thiamine treated OLETF rats in this study. Metabolic parameters (glucose, triglyceride and total cholesterol) in LETO-Breeder and OLETF-Breeder were obtained from fed blood samples. Metabolic parameters in OLETF-control and OLETF-Thiamine were obtained from 16-h fasting blood samples. 
c

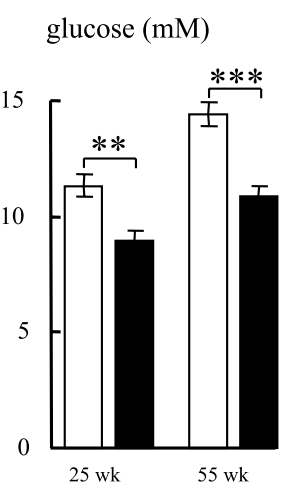

insulin (nM)

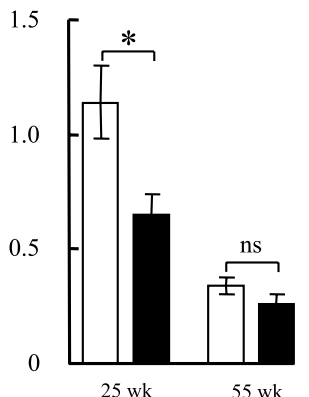

$\mathrm{Hb} \mathrm{A} 1 \mathrm{C}(\%)$

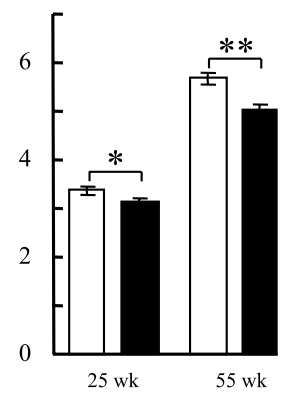

TG (mM)

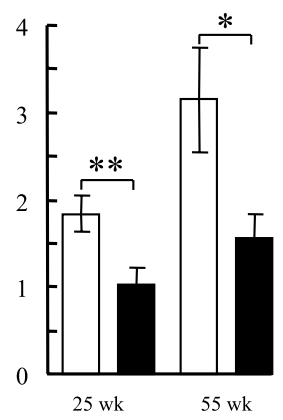

T-Cho (mM)

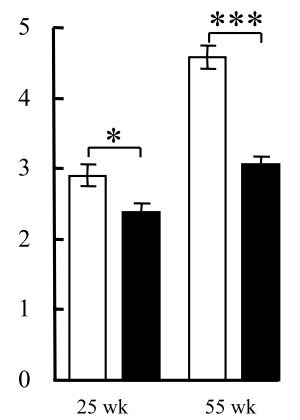

NEFA (mM)

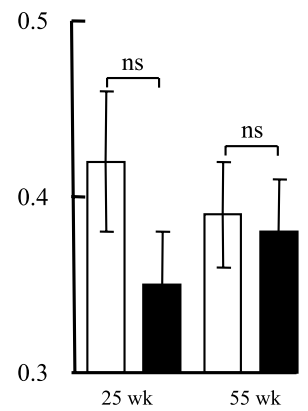

ketone (mM)

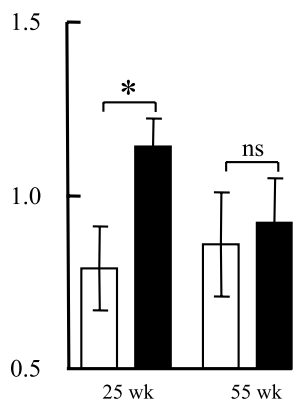

Fig. 2. Thiamine averted obesity-associated metabolic disorders in OLETF rats. Blood was obtained from OLETF rats provided water containing $0 \%$ or $0.2 \%$ thiamine after $16 \mathrm{~h}$ of fasting. Bar graphs indicate serum glucose, insulin, glycated haemoglobin (HbA1c), triglycerides (TGs), total cholesterol (T-Cho), non-esterified fatty acids (NEFAs) and ketone bodies. Open columns indicate control OLETF rats. Closed columns indicate thiamine-treated OLETF rats. Bars indicate means \pm SE for $n=8 .{ }^{*} p<0.05$; ${ }^{* *} p<0.01{ }^{* * *} p<0.001$ by an unpaired, two-tailed Student's $t$ test. ns, not significant.

ferences were considered significant at $p<0.05$.

\section{RESULTS AND DISCUSSION}

Thiamine decreased body and organ weights of OLETF rats

OLETF rats ( $4 \mathrm{wk}$ old) were given water (control group) or water containing $0.2 \%$ thiamine (thiamine group) for $21 \mathrm{wk}(n=16)$ and $51 \mathrm{wk}(n=8)$ in each group. No difference in body weight was observed between the two groups at the start of this study $(62.7 \pm 1.9 \mathrm{~g}$ in the control and $66.2 \pm 1.3 \mathrm{~g}$ in the thiamine group; $p=0.13)$. Food consumption relative to body weight in the thiamine group was equivalent to that in the control group throughout the experimental period. Body weight gain in the thiamine group was significantly less than that in the control group at $4 \mathrm{wk}$ after treatment and, at $55 \mathrm{wk}$ of age, thiamine decreased body weight by over 20\% (Fig. 1a). Unfortunately, in this study, we did not assess Long-Evans Tokushima Otsuka (LETO) rats, nondiabetic/lean counterparts of OLETF rats. For reference, body weights in breeder's data sheet are depicted in Table 1. The decreased body weight with thiamine treatment did not reach the levels of LETO rats.

Figure $1 \mathrm{~b}$ illustrates general physiological characteristics of control and thiamine-treated OLETF rats. Consistent with the known lack of toxicity to thiamine, we observed no adverse effects in the thiamine group. Equivalent tibia lengths were observed in both groups, suggesting no substantial growth retardation in the thiamine group. The thiamine group was observed to have lowered weights of the heart, liver, kidney and epididymal and retroperitoneal fat pads. Among these, weights of retroperitoneal fat pads of rats at 21 and 51 wk of treatment were approximately 76 and $60 \%$ of that of control group rats, respectively, suggesting that reduced accumulation of fat most likely accounted for the weight difference in thiamine-treated OLETF rats. Cancer ( 1 in the kidney and 1 in the spleen) was detected in 2 control OLETF rats but not in thiamine-treated OLETF rats.

Thiamine averted obesity-associated metabolic disorders in OLETF rats

Figure 2 graphically depicts blood chemistries of control and thiamine-treated OLETF rats obtained after $16 \mathrm{~h}$ of fasting. The control group had higher levels of serum glucose, insulin, HbA1c, TG and T-Cho. Thiamine treatment significantly reduced these metabolic parameters, indicating the deterrence of insulin resistance and dyslipidemia in thiamine-treated OLETF rats. Metabolic parameters obtained from fed blood samples on the breeder's data sheet are depicted in Table 1 for reference; however metabolic parameters in this study were evaluated in 16-h fasting blood samples. Thus, values could not be directly compared to each other. We only show them for information.

Total ketone bodies in thiamine treated OLETF rats 
a

control

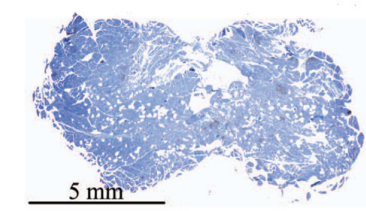

thiamine-treated

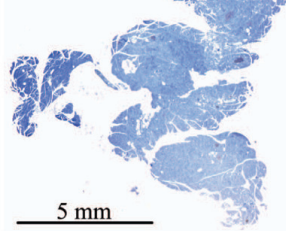

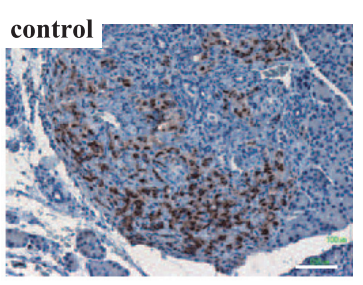

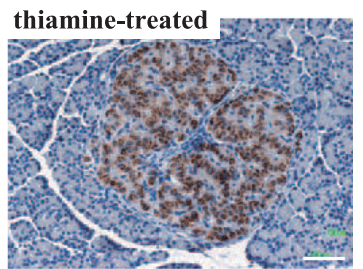

b
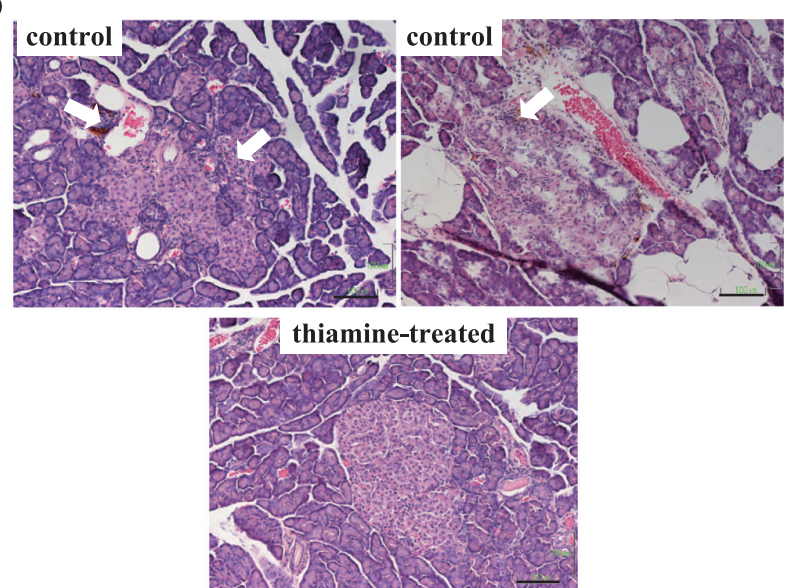

Fig. 4. a, b.

a

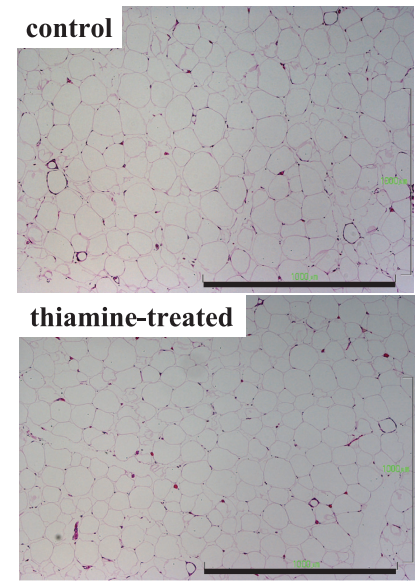

Fig. 3. a, b.

b

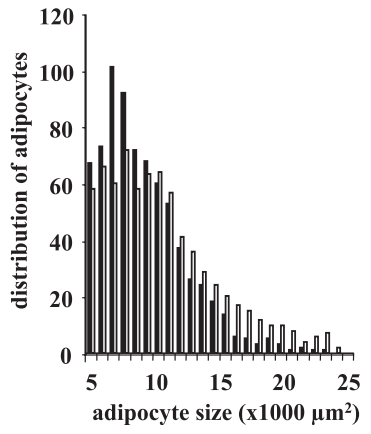

a

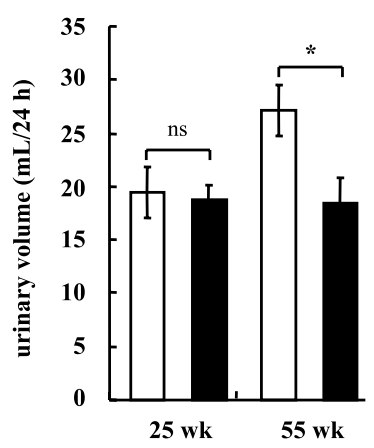

b
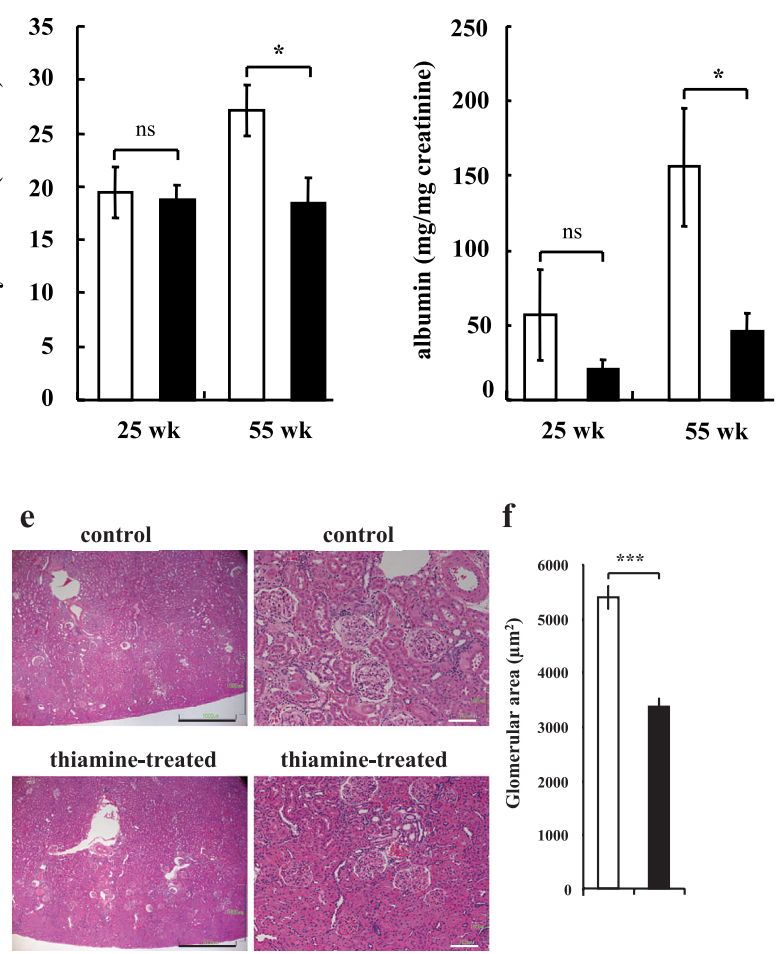

f

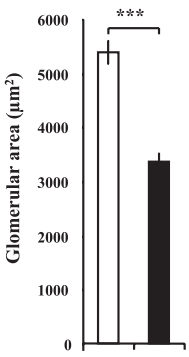

i
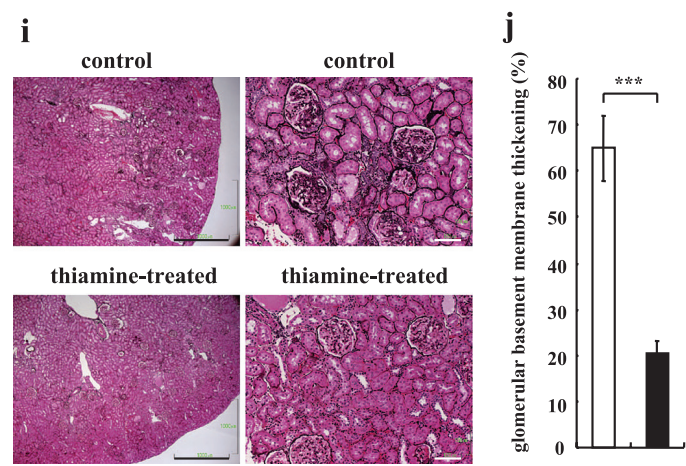

Fig. 5. a-j. 
a

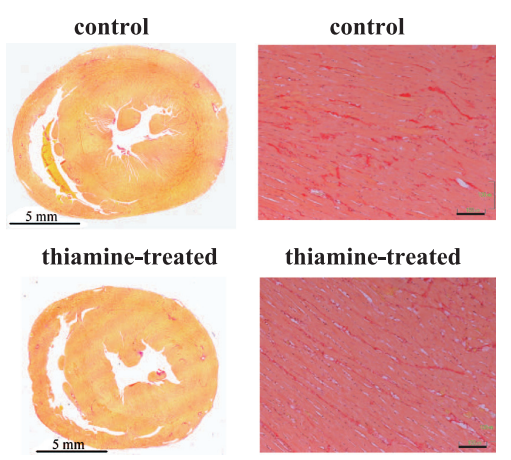

b

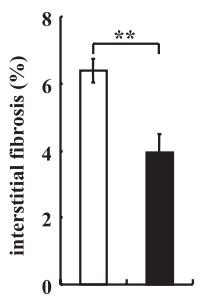

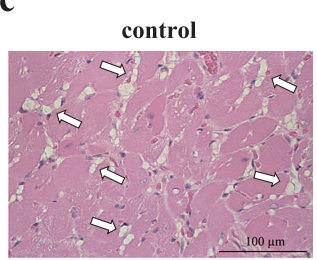

thiamine-treated

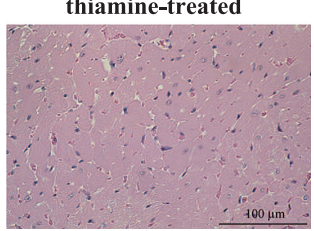

d

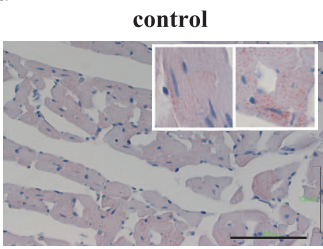

thiamine-treated

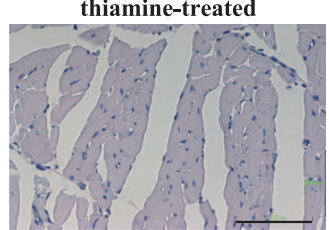

Fig. 6. a-d.

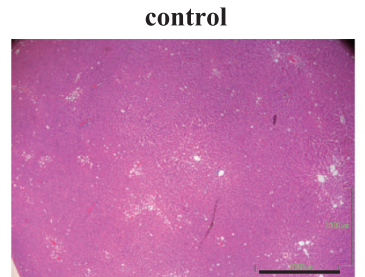

thiamine-treated

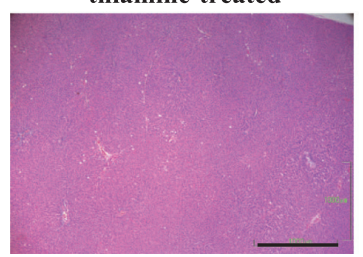

c

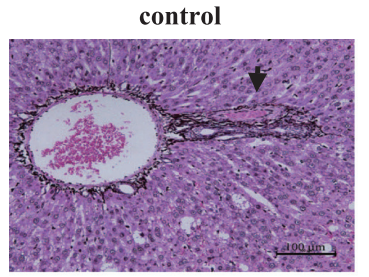

thiamine-treated

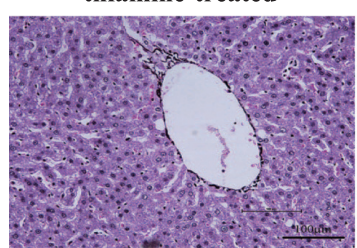

control

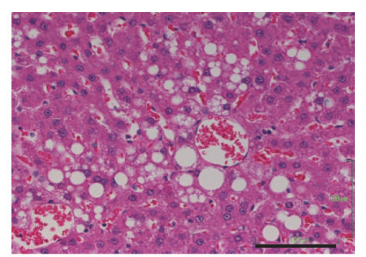

thiamine-treated

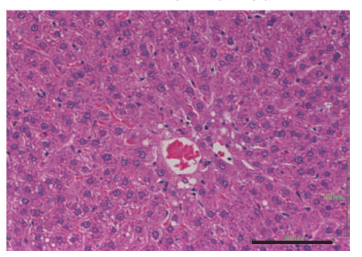

control

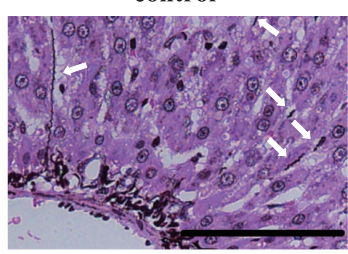

thiamine-treated

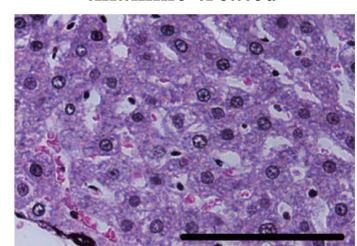

b

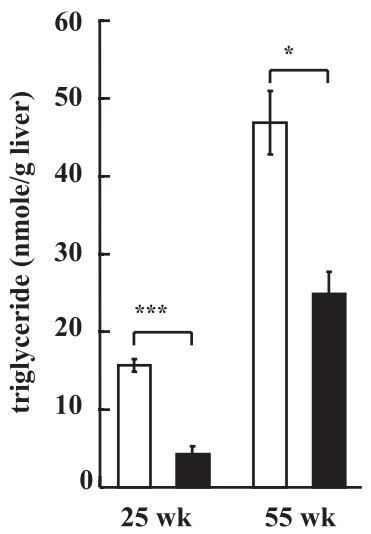

d

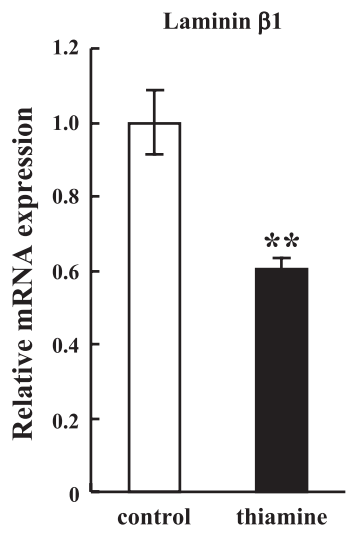

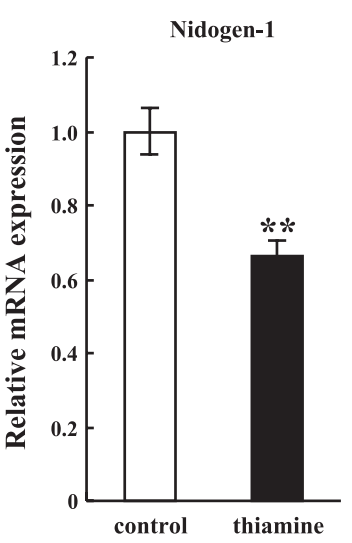

Fig. 7. a-d.

were higher than those of untreated OLETF rats. PiSunyer has reported the increase of total ketone bodies in fasted Sprague-Dawley male rats $(0.398 \mathrm{~mm}$ in feeding rats vs. $1.143 \mathrm{~mm}$ in 24-h fasting rats) (25). Total ketone bodies in thiamine-treated OLETF rats of $25 \mathrm{wk}$ old (1.14 $\pm 0.08 \mathrm{mM}$ in 16 -h fasting) were comparable to those of Pi-Sunyer's report. Studies have indicated that lipid oxidation is reduced in obese human skeletal muscles, and decreased reliance on lipids as an energy source has been identified as a metabolic risk factor for weight gain $(26,27)$. Lower total ketone bodies in untreated OLETF rats may suggest reduced lipid oxidation, which was mitigated by thiamine.
Thiamine diminished adipocyte size in OLETF rats

Thiamine treatment profoundly decreased visceral fat mass in OLETF rats (Fig. 1b). Lipid accumulation in visceral white adipose tissue is considered a cause of obesity and obesity-associated metabolic disorders. The size of adipocytes is known to influence their function and change in the large adipocyte subfraction has been suggested to contribute to the improvement in insulin sensitivity (28). We therefore evaluated the size of epididymal adipocytes.

Photographs of epididymal white adipose tissues obtained from control and thiamine-treated OLETF rat at $55 \mathrm{wk}$ of age as well as histograms of adipose cell 
sizes are represented in Fig. 3a. Histological evaluations revealed that decreasing of numbers in the large adipocyte subfraction in epididymal white adipose tissues were predominant in the thiamine-treated group compared to the control group (Fig. 3b).

Thiamine modified the function and morphology in pancreases of OLETF rats

Obesity frequently accompanies insulin resistance. Thiamine treatment significantly modified glycemic controls in OLETF rats (Fig. 2).

Histological evaluations of pancreases disclosed the following findings. At $25 \mathrm{wk}$ of age, morphologies of pancreases, except for the islets, in both groups appeared normal (data not shown). The islets of control OLETF rats were enlarged compared to those of thia- mine-treated OLETF rats. At $55 \mathrm{wk}$ of age, numerous vacuoles, suggesting lipid, were observed in pancreases of control OLETF rats (Fig. 4a). The islets of the control group were disorganized. Insulin-positive cells were atrophied and few in number, and the contours of the islets were irregular and could not be easily delineated (Fig. 4a). H\&E staining revealed numerous focal regions of degeneration and mononuclear cell infiltration (indicated by white arrows) in the islets of control OLETF rats (Fig. 4b). In contrast, pancreases of the thiaminetreated group had minimal lipid droplet accumulations, mild mononuclear cell infiltrations and well-preserved islet appearances. These histological results, together with biochemical results, suggested that OLETF rats reflected a hyperinsulinemic state at $25 \mathrm{wk}$ of age and a

Fig. 3. Thiamine diminished adipocyte size in OLETF rats. a: Sections of epididymal white adipose tissue obtained from 55wk-old OLETF rats were stained with hematoxylin and eosin (H\&E) (bar=1,000 $\mu \mathrm{m}$ ). b: The histogram illustrates the size distribution of adipocytes and indicates the predominance of smaller adipocytes in the thiamine group. Sections of epididymal adipose tissue were obtained from 6 control and 6 thiamine-treated OLETF rats. Open columns indicate control OLETF rats. Closed columns indicate thiamine-treated OLETF rats.

Fig. 4. Thiamine attenuated fatty degeneration in pancreases of OLETF rats. a: Pancreases obtained from OLETF rats at $55 \mathrm{wk}$ of age were stained with anti-insulin antibody and counter-stained with haematoxylin. The left column shows whole a pancreas specimen ( $\mathrm{bar}=5 \mathrm{~mm}$ ). The right column shows microscopic findings (bar=100 $\mu \mathrm{m})$. b: Photomicrographs stained with H\&E (bar $=100 \mu \mathrm{m})$. White arrows indicate mononuclear cell infiltration.

Fig. 5. Thiamine modified function and morphology of kidneys in OLETF rats. a: Urinary volume. Urines were collected for $24 \mathrm{~h}$ from rats, at $55 \mathrm{wk}$ of age, kept in individual metabolic cages. Open columns indicate control OLETF rats. Closed columns indicate thiamine-treated OLETF rats. Bars indicate means \pm SE for $n=6 .{ }^{*} p<0.05$ by an unpaired, two-tailed Student's $t$ test. ns, not significant. b: Urinary albumin excretion. Urinary albumin levels were expressed as albumin-to-creatinine ratios. Open columns indicate control OLETF rats. Closed columns indicate thiamine-treated OLETF rats. Bars indicate means \pm SE for $n=6 .{ }^{*} p<0.05$ by an unpaired, two-tailed Student's $t$ test. ns, not significant. c: Kidneys obtained from OLETF rats at $55 \mathrm{wk}$ of age were stained with Picric Acid Sirius Red for evaluation of fibrosis. The left column shows whole kidneys (bar $=5 \mathrm{~mm}$ ). The right column shows microscopic findings (bar $=100 \mu \mathrm{m})$. d: Quantitative evaluation of renal interstitial fibrosis. The open column indicates control OLETF rats. The closed column indicates thiamine-treated OLETF rats. Bars indicate mean \pm SE for $n=6 .{ }^{* * *} p<0.001$. e: H\&E staining. The left column shows low magnification images $(\mathrm{bar}=1,000 \mu \mathrm{m}$ ). The right column shows high magnification images (bar $=100 \mu \mathrm{m})$. f: Quantitative evaluation of glomerular size. The open column indicates control OLETF rats. The closed column indicates thiamine-treated OLETF rats. Bars indicate means \pm SE for $n=6$. ${ }^{* * *} p<0.001$. g: PAS stain. The left column shows low magnification images (bar $=1,000 \mu \mathrm{m}$ ). The right column shows high magnification images (bar $=100 \mu \mathrm{m})$. White arrows indicate mononuclear cell infiltrations. h: Quantitative evaluation of glomerular sclerosis. The open column indicates control OLETF rats. The closed column indicates thiamine-treated OLETF rats. Bars indicate means \pm SE for $n=6 .{ }^{* * *} p<0.001$. i: PAM stain. The left column shows low magnification images (bar $=1,000 \mu \mathrm{m}$ ). The right column shows high magnification images (bar=100 $\mu \mathrm{m}$ ). j: Quantitative evaluation of the thickening of the glomerular basement membrane. The open column indicates control OLETF rats. The closed column indicates thiamine-treated OLETF rats. Bars indicate means \pm SE for $n=6$. **** $p<0.001$.

Fig. 6. Thiamine evaded interstitial fibrosis and steatosis in hearts of OLETF rats. a: Hearts obtained from OLETF rats at 55 wk of age were stained with Picric Acid Sirius Red for evaluation of fibrosis. The left column shows an image of the whole heart (bar $=5 \mathrm{~mm}$ ). The right column shows microscopic findings (bar $=100 \mu \mathrm{m}$ ). b: Quantitative evaluation of cardiac interstitial fibrosis. The open column indicates control OLETF rats. The closed column indicates thiamine-treated OLETF rats. Bars indicate means \pm SE for $n=6$. ${ }^{* * *} p<0.01$. c: H\&E staining (bar $\left.=100 \mu \mathrm{m}\right)$. White arrows indicate vacuoles, most likely indicating lipid droplets. d: Oil Red O staining (bar $=100 \mu \mathrm{m})$. Inserts in control OLETF rat are higher magnification images. Red granules, indicating lipids, are robust in control OLETF rat's heart.

Fig. 7. Thiamine decreased hepatic TG accumulation and modulated hepatic vasculatures in OLETF rats. a: H\&E stained sections of livers from 55-wk-old OLETF rats. The left column shows low magnification images (bar=1 mm). The right column shows high magnification images (bar=100 $\mu \mathrm{m}$ ). A photomicrograph from the control OLETF rat at high magnification shows robust macrovesicular steatosis, which is reduced with thiamine intervention. b: Quantitative evaluation of TG contents in livers of OLETF rats. Open columns indicate control OLETF rats. Closed columns indicate thiamine-treated OLETF rats. Bars indicate means \pm SE for $n=6 .{ }^{*} p<0.05 ;{ }^{* * *} p<0.001$. c: Periodic acid-methenamine-silver (PAM) stain of livers from 55-wk-old OLETF rats. The left column shows low magnification images (bar=100 $\mu \mathrm{m}$ ). The black arrow indicates a vascular architecture. A higher magnification view (right column) shows components stained with PAM along sinusoids (white arrows; bar $=100 \mu \mathrm{m}$ ). d: Effect of thiamine on laminin $\beta 1$ and nidogen- 1 mRNA expression in liver of OLETF rats. Total RNA was extracted from the liver and real-time RT-PCR analysis was performed. Columns show relative mRNA expression of laminin $\beta 1$ and nidogen-1. Open columns indicate control OLETF rats. Closed columns indicate thiamine-treated OLETF rats. Bars indicate means \pm SE for $n=6 .{ }^{*} p<0.005$. 
diabetic state, resulting from the islets destruction, at $55 \mathrm{wk}$ of age. Thus, thiamine prevented development of obesity-associated insulin resistance and diabetes in OLETF rats.

Thiamine mitigated albuminuria, renal interstitial fibrosis, glomerulomegaly, basement membrane thickening and focal glomerulosclerosis in OLETF rats

Obesity and type 2 diabetes are risk factors for the development of chronic kidney disease, which is frequently accompanied by proteinuria and albuminuria. Kidney weights were significantly reduced in thiaminetreated OLETF rats at $55 \mathrm{wk}$ of age (Fig. 1b). Thiamine treatment significantly reduced urinary volume and urinary albumin excretion in OLETF rats at $55 \mathrm{wk}$ of age (Fig. 5b).

Renal lesions in OLETF rats were characterized by thickening of the glomerular basement membrane, mesangial expansion, sclerotic lesions, interstitial fibrosis (29) and interstitial lymphocyte infiltration (30). In this study, histological appearances revealed findings comparable to these observations. Images of kidneys from OLETF rats at $55 \mathrm{wk}$ of age stained with Picric Acid Sirius Red, H\&E, PAS and PAM are represented in Fig. 5c, e, g and i, respectively.

Thiamine treatment affected the renal architecture in OLETF rats as follows: (1) Significantly reduced interstitial fibrosis. Renal interstitial fibrosis, as assessed by Picric Acid Sirius Red, was decreased by approximately 65\% (Fig. 5d). (2) Significantly reduced glomerulomegaly. Glomerular size, assessed by the glomerular area, was decreased by approximately 40\% (Fig. 5f). (3) Significantly diminished focal glomerular sclerosis. The degree of focal glomerular sclerosis, as assessed by PAS positive staining of more than $50 \%$ of the glomerulus, was reduced by approximately 55\% (Fig. 5h). (4) Significantly decreased thickening of the glomerular basement membrane. Thickening of the glomerular basement membrane and deposition of PAM-positive ECM in the glomerulus, which was notable in control OLETF rats at $55 \mathrm{wk}$ of age, were milder in thiamine-treated OLETF rats at $55 \mathrm{wk}$ of age. The relative amount of glomerular basement membrane thickening, as assessed by robust staining with PAM, was decreased by approximately 70\% (Fig. 5j). (5) Interstitial lymphocyte infiltration was largely attenuated by thiamine treatment (Fig. 5g, indicated by white arrows). These results signify preventive effects of thiamine on both renal architectural and functional abnormalities in OLETF rats.

Thiamine preserved cardiac function and circumvented interstitial fibrosis and steatosis in hearts of OLETF rats

The heart weights were significantly lower after 51 wk of thiamine treatment (Fig. 1b). Systolic blood pressures of thiamine-treated OLETF rats, at $55 \mathrm{wk}$ of age, were significantly lower than those of control OLETF rats (Table 2).

Studies in humans and animals suggest that obesity may be associated with abnormally high levels of TGs in the heart. Adiposity directly damages the heart by promoting ectopic deposition of TG, a process known as
Table 2. Effects of thiamine on echocardiography parameters in OLETF rats at $55 \mathrm{wk}$ of age.

\begin{tabular}{lrrr}
\hline & \multicolumn{1}{c}{$\begin{array}{c}\text { Control } \\
(n=6)\end{array}$} & \multicolumn{1}{c}{$\begin{array}{c}\text { Thiamine } \\
(n=6)\end{array}$} & \multicolumn{1}{c}{$p$} \\
\hline SBP $(\mathrm{mmHg})$ & $139.25 \pm 2.61$ & $131.08 \pm 1.84$ & 0.023 \\
Heart rate & $398.55 \pm 3.55$ & $391.28 \pm 6.01$ & 0.315 \\
Dd (mm) & $9.37 \pm 0.12$ & $8.57 \pm 0.13$ & 0.001 \\
Ds (mm) & $5.15 \pm 0.16$ & $4.07 \pm 0.17$ & $<0.001$ \\
IVSd (mm) & $1.52 \pm 0.05$ & $1.44 \pm 0.03$ & 0.228 \\
LV Pwd (mm) & $1.47 \pm 0.07$ & $1.44 \pm 0.05$ & 0.730 \\
h (mm) & $1.47 \pm 0.04$ & $1.45 \pm 0.04$ & 0.748 \\
FS (\%) & $44.69 \pm 1.08$ & $52.48 \pm 1.60$ & 0.002 \\
LVEF $(\%)$ & $83.00 \pm 0.01$ & $89.00 \pm 0.01$ & 0.003 \\
E/A ratio & $1.26 \pm 0.07$ & $1.76 \pm 0.11$ & 0.002 \\
DecT (ms) & $41.67 \pm 2.77$ & $59.33 \pm 2.39$ & $<0.001$ \\
E/Ea ratio & $27.91 \pm 1.53$ & $18.93 \pm 0.87$ & $<0.001$ \\
\hline
\end{tabular}

SBP, systolic blood pressure; LV, left ventricular; Dd, enddiastolic diameter of LV cavity; Ds, end-systolic diameter of LV cavity; FS, fractional shortening; IVSd, interventricular septum dimension at diastole; PWd, posterior wall dimension at diastole; h, mean of IVSd and PWd; LVEF, $\mathrm{LV}$ ejection fraction; E, early diastolic flow; A, atrial contraction flow; DecT, deceleration time of E; Ea, the early diastolic velocity recorded at the lateral corner of the annulus.

myocardial steatosis. Cardiac steatosis can lead to dilated cardiomyopathy and an impaired cardiac function, so-called lipotoxic cardiomyopathy (31-33). Accordingly, cardiac functions were assessed by echocardiography in 55-wk-old OLETF rats.

Echocardiograms revealed that thiamine treatment resulted in the following: (1) Decreased left ventricular (LV) cavity size assessed by Dd and Ds. (2) Preserved systolic function assessed by LVEF and FS. (3) Preserved diastolic function assessed by early diastolic flow (E)/ atrial contraction flow (A) ratio, deceleration time of early diastolic flow, and E/Ea (Table 2). LV wall thickness, represented by $\mathrm{h}$, was equivalent in both groups (Table 2). These findings indicated systolic and diastolic dysfunctions in control OLETF rats, which were attenuated by thiamine intervention.

Myocardial tissue sections were stained with Picric Acid Sirius Red to determine interstitial fibrosis and with Oil Red $\mathrm{O}$ to determine intramyocardial neutral lipid accumulation in control OLETF rats and thiaminetreated OLETF rats at $55 \mathrm{wk}$ of age. Picric Acid Sirius Red staining revealed an approximately $40 \%$ decrease of interstitial fibrosis with thiamine treatment (Fig. 6b). H\&E staining revealed vacuoles, most likely lipid droplets, which were prominent in control OLETF rats compared to thiamine-treated OLETF rats (Fig. 6c). Oil Red $O$ staining revealed reduced positive staining in hearts of thiamine-treated OLETF rats compared to that in control OLETF rats (Fig. 6d). Oil Red O staining also indicated reduced positive staining in gastrocnemius muscles of thiamine-treated OLETF rats compared to control OLETF rats (data not shown). Taken together, thiamine abrogated TG accumulation in the heart and 
averted cardiac dysfunctions in OLETF rats.

Thiamine decreased hepatic TG accumulation, modulated hepatic vasculatures and increased hepatic active PDH activity in OLETF rats

Thiamine treatment significantly reduced liver weights of OLETF rats (Fig. 1b). Hepatic steatosis is a common pathological condition of obesity and diabetes mellitus. Representative images of liver sections from OLETF rats at $55 \mathrm{wk}$ of age stained with H\&E are shown in Fig. 7a. Vacuoles, most likely lipid droplets, were prominent in control OLETF rats compared to thiaminetreated OLETF rats. Reduced hepatic steatosis with thiamine treatment was supported by the significant attenuation in hepatic TG accumulation (Fig. 7b).

Additionally, hepatic microangiopathy, although not widely recognized, is reported to be associated with diabetes mellitus. Thickening of capillary basement membranes in vascular beds is a hallmark of microangiopathy. Normal hepatic sinusoids, unlike other vascular beds, have no basement membranes. Sinusoidal fibrosis with formation of basement membranes (called pseudocapillarization), which may contribute to impaired clearance of chylomicron remnants, postprandial hypertriglyceridemia and atherosclerosis (34), was observed in diabetic patients (35). When analyzed by immunostaining, basement membrane components of pseudocapillarization were reported to be similar to those observed in kidneys in diabetic glomerulosclerosis cases (36). Hence, hepatic vasculature morphologies were also assessed using PAM stain, which revealed basement membrane thickening in the central vein, and hepatic artery and pseudocapillarization in control OLETF rats (Fig. 7c). Findings of hepatic artery thickening correlate with arteriopathy that is known to occur in other organs in cases of diabetes mellitus. PAM-positive staining of hepatic vasculatures was not robust in thiamine-treated OLETF rats. Effects of thiamine on PAM findings in the liver showed impressive similarities with findings in the glomerulus.

Basement membranes are organized with several components. A ubiquitous basement membrane protein, nidogen-1, is encoded by Nid1, and another basement membrane protein, laminin $\beta 1$, is encoded by Lamb1. Profiles of gene transcript expressions in livers of control and thiamine-treated OLETF rats using gene microarrays indicated that, of the 33,849 genes on the array, $76(0.22 \%$ of total genes on the array) showed 2fold or greater differences of expression in the thiaminetreated rats compared to control group rats (data not shown. Microarray data and experimental details were submitted to GEO. http://www.ncbi.nlm.nih.gov/geo/ query/acc.cgi?acc=GSE19292, http://www.ncbi.nlm. nih.gov/geo/query/acc.cgi?acc=GSM476602, http:// www.ncbi.nlm.nih.gov/geo/query/acc.cgi?acc $=$ GSM47 6603, http://www.ncbi.nlm.nih.gov/geo/query/acc. cgi?acc $=$ GSM476604, http://www.ncbi.nlm.nih.gov/ geo/query/acc.cgi?acc $=$ GSM476605). Among these 76 genes, Lamb1 and Nid1 were decreased in the liver of thiamine-treated OLETF rats.

Laminin expression was reported to be frequent in

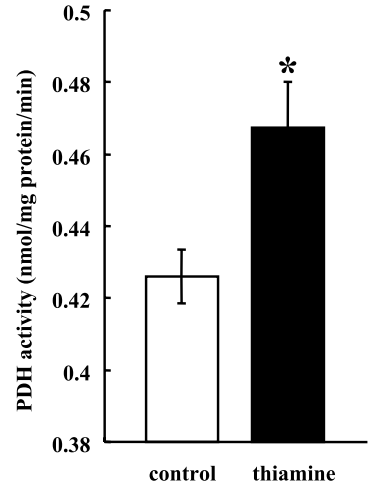

Fig. 8. Thiamine increased hepatic active PDH activity in OLETF rats. Pyruvate dehydrogenase (PDH) activity in hepatic mitochondrial fraction. Mitochondrial fractions were prepared from OLETF rats' livers at $55 \mathrm{wk}$ of age. Open columns indicate control OLETF rats. Closed columns indicate thiamine-treated OLETF rats. Values are mean \pm SE for $n=4$ control and 7 thiamine-treated rats. ${ }^{*} p<0.05$.

livers of diabetic patients, but not in normal livers (35). Nidogen-1 interacts with many other basement membrane components, particularly laminin and collagen IV, and implies an essential integrating role of basement membrane assembly $(37,38)$. Accordingly, real-time RT-PCR analysis was performed to assess the expression of laminin $\beta 1$ and nidogen-1 mRNA. The mRNA expression of laminin $\beta 1$ and nidogen- 1 in livers was significantly depressed in thiamine-treated OLETF rats compared to untreated OLETF rats (Fig. 7d). Lowered transcript expressions of laminin $\beta 1$ and nidogen- 1 genes underpinned hepatic, and perhaps also renal, morphological findings that revealed reduced basement membrane thickening in thiamine-treated OLETF rats. Thus, thiamine intervention modulated hepatic TG accumulation as well as hepatic vasculatures in OLETF rats.

Reduced PDH activity in OLETF rats was reported (39), so we measured active PDH activity in the liver of OLETF rats. Thiamine repletion significantly increased active PDH activity in the liver of OLETF rats (Fig. 8).

$\mathrm{PDH}$ complex contributes to transforming pyruvate into acetyl-CoA, which may then be used in the citric acid cycle. Thus, PDH is the key enzyme in glucose oxidative breakdown.

Alterations in intracellular glucose metabolism have been revealed in diabetes mellitus. For glucose-mediating cellular damage Brownlee's group has proposed the integrating paradigm that is the activation of four minor pathways in glucose metabolism. In addition to oxidation, glucose could be shunted into at least four minor pathways, e.g., the hexosamine biosynthetic pathway, the polyol pathway, the diacylglycerol pathway, and the methylglycerol pathway; all of which have been suggested to be stimulated with the increased flux of glucose (4).

Increased availability of the glycolytic metabolites glyceraldehyde-3-phosphate and fructose-6-phosphate could activate the hexosamine biosynthetic pathway, 
the diacylglycerol pathway, and the methylglycerol pathway. The lipid-soluble thiamine derivative benfotiamine has been shown to inhibit these three pathways, as well as hyperglycemia-associated NF- $\kappa \mathrm{B}$ activation, by activating the pentose phosphate pathway enzyme transketolase, which converts glyceraldehyde3-phosphate and fructose-6-phosphate into pentose-5phosphates and other sugars (6).

PDH contributes to linking the glycolysis metabolic pathway to the citric acid cycle, which converts acetylCoA into ATP, NADH and carbon dioxide. PDH activity has been suggested to be significantly below normal in the circulating lymphocytes of obese nondiabetic subjects as well as obese and nonobese newly diagnosed non insulin dependent diabetes mellitus patients (40). $\mathrm{PDH}$ and transketolase require thiamine as a coenzyme. Thus, activation of PDH, similar to activated transketolase activity with benfotiamine (6), could also reduce increased availability of the glycolytic metabolites and could avert the activation of minor pathways in glucose metabolism in OLETF rats.

Closing notes

Obesity and its related metabolic disorders are at epidemic proportions and show no signs of reduction in incidence. Many factors, including genetics, environment and lifestyle changes, undoubtedly provide impetus to an increase in these morbidities. Among these factors, we believe that changes in dietary habits should be given considerable importance when dealing with these pandemic morbidities.

Modern diets largely comprise processed foods high in carbohydrates and fats, which are cheap and easily available in supermarkets and fast-food restaurants. However, techniques used for high refining of grains can lead to loss of micronutrients, including thiamine. Liu and Manson suggested that replacing high refined grain products with minimally processed plant-based foods, such as whole grains, may offer a simple strategy for reducing the incidence of coronary heart disease (41).

Current pharmaceutical interventions are moderately effective in treating clinical symptoms of metabolic syndrome; nevertheless, a fundamental pharmaceutical arm for preventing obesity and its related metabolic disorders remains to be established. Although additional corroboration is necessary, present findings indicate that thiamine may be beneficial for targeting composite physiological abnormalities, rather than individual component criteria, and could be utilized for preventive intervention. Given the detrimental effects of obesity and the safety and cost effectiveness of thiamine, we believe that thiamine offers little to lose and much to gain.

\section{Acknowledgments}

This study was partly supported by the High Technology Research Center Project for Private Universities: matching fund subsidy from the Ministry of Education, Culture, Sports, Science, and Technology of Japan and by the Research Grant for Cardiovascular Disease from the Ministry of Health, Labor, and Welfare. OLETF male rats were kindly supplied by the Tokushima Research Institute, Otsuka Pharmaceutical (Tokushima, Japan).

\section{REFERENCES}

1) Moller DE, Kaufman KD. 2005. Metabolic syndrome: a clinical and molecular perspective. Annu Rev Med 56: 45-62.

2) WHO. 2000. Obesity: preventing and managing the global epidemic. Report of a WHO consultation. World Health Organ Tech Rep Ser 894: 1-253.

3) Babaei-Jadidi R, Karachalias N, Ahmed N, Battah S, Thornalley PJ. 2003. Prevention of incipient diabetic nephropathy by high-dose thiamine and benfotiamine. Diabetes 52: 2110-2120.

4) Brownlee M. 2001. Biochemistry and molecular cell biology of diabetic complications. Nature 414: 813820.

5) Ceylan-Isik AF, Wu S, Li Q, Li SY, Ren J. 2006. High-dose benfotiamine rescues cardiomyocyte contractile dysfunction in streptozotocin-induced diabetes mellitus. $J$ Appl Physiol 100: 150-156.

6) Hammes HP, Du X, Edelstein D, Taguchi T, Matsumura T, Ju Q, Lin J, Bierhaus A, Nawroth P, Hannak D, Neumaier M, Bergfeld R, Giardino I, Brownlee M. 2003. Benfotiamine blocks three major pathways of hyperglycemic damage and prevents experimental diabetic retinopathy. Nat Med 9: 294-299.

7) Kohda Y, Shirakawa H, Yamane K, Otsuka K, Kono T, Terasaki F, Tanaka T. 2008. Prevention of incipient diabetic cardiomyopathy by high-dose thiamine. J Toxicol Sci 33: 459-472.

8) Stirban A, Negrean M, Stratmann B, Gawlowski T, Horstmann T, Götting C, Kleesiek K, Mueller-Roesel M, Koschinsky T, Uribarri J, Vlassara H, Tschoepe D. 2006. Benfotiamine prevents macro- and microvascular endothelial dysfunction and oxidative stress following a meal rich in advanced glycation end products in individuals with type 2 diabetes. Diabetes Care 29: 2064-2071.

9) Stracke H, Hammes HP, Werkmann D, Mavrakis K, Bitsch I, Netzel M, Geyer J, Köpcke W, Sauerland C, Bretzel RG, Federlin KF. 2001. Efficacy of benfotiamine versus thiamine on function and glycation products of peripheral nerves in diabetic rats. Exp Clin Endocrinol Diabetes 109: 330-336.

10) Stracke H, Lindemann A, Federlin KF. 1996. A benfotiamine-vitamin B combination in treatment of diabetic polyneuropathy. Exp Clin Endocrinol Diabetes 104: 311316.

11) Abboud MR, Alexander D, Najjar SS. 1985. Diabetes mellitus, thiamine-dependent megaloblastic anemia, and sensorineural deafness associated with deficient $\alpha$ ketoglutarate dehydrogenase activity. J Pediatr 107: 537-541.

12) Alzahrani A, Baitei E, Zou M, Shi Y. 2006. Thiamine transporter mutation: an example of monogenic diabetes mellitus. Eur J Endocrinol 155: 787-792.

13) Borgna-Pignatti C, Marradi P, Pinelli L, Monetti N, Patrini C. 1989. Thiamine-responsive anemia in DIDMOAD syndrome. J Pediatr 114: 405-410.

14) Neufeld EJ, Fleming JC, Tartaglini E, Steinkamp MP. 2001. Thiamine-responsive megaloblastic anemia syndrome: a disorder of high-affinity thiamine transport. Blood Cells Mol Dis 27: 135-138.

15) Elbein SC, Hoffman MD, Teng K, Leppert MF, Hasstedt 
SJ. 1999. A genome-wide search for type 2 diabetes susceptibility genes in Utah Caucasians. Diabetes $\mathbf{4 8}$ : 1175-1182.

16) Hanson RL, Ehm MG, Pettitt DJ, Prochazka M, Thompson DB, Timberlake D, Foroud T, Kobes S, Baier L, Burns DK, Almasy L, Blangero J, Garvey WT, Bennett PH, Knowler WC. 1998. An autosomal genomic scan for loci linked to type II diabetes mellitus and body-mass index in Pima Indians. Am J Hum Genet 63: 11301138.

17) Thornalley PJ, Babaei-Jadidi R, Al Ali H, Rabbani N, Antonysunil A, Larkin J, Ahmed A, Rayman G, Bodmer CW. 2007. High prevalence of low plasma thiamine concentration in diabetes linked to a marker of vascular disease. Diabetologia 50: 2164-2170.

18) Valerio G, Franzese A, Poggi V, Patrini C, Laforenza U, Tenore A. 1999. Lipophilic thiamine treatment in longstanding insulin-dependent diabetes mellitus. Acta Diabetol 36: 73-76.

19) Aitman TJ, Gotoda T, Evans AL, Imrie H, Heath KE, Trembling PM, Truman H, Wallace CA, Rahman A, Doré C, Flint J, Kren V, Zidek V, Kurtz TW, Pravenec M, Scott J. 1997. Quantitative trait loci for cellular defects in glucose and fatty acid metabolism in hypertensive rats. Nat Genet 16: 197-201.

20) Aitman TJ, Glazier AM, Wallace CA, Cooper LD, Norsworthy PJ, Wahid FN, Al-Majali KM, Trembling PM, Mann CJ, Shoulders CC, Graf D, St-Lezin E, Kurtz TW, Kren V, Pravenec M, Ibrahimi A, Abumrad NA, Stanton LW, Scott J. 1999. Identification of Cd36 (Fat) as an insulin-resistance gene causing defective fatty acid and glucose metabolism in hypertensive rats. Nat Genet $\mathbf{2 1}$ : 76-83.

21) Tanaka T, Sohmiya K, Kono T, Terasaki F, Horie R, Ohkaru Y, Muramatsu M, Takai S, Miyazaki M, Kitaura Y. 2007. Thiamine attenuates the hypertension and metabolic abnormalities in CD36-defective SHR: uncoupling of glucose oxidation from cellular entry accompanied with enhanced protein O-GlcNAcylation in CD36 deficiency. Mol Cell Biochem 299: 23-35.

22) Bligh EG, Dyer WJ. 1959. A rapid method of total lipid extraction and purification. Can J Biochem Physiol 37: 911-917.

23) Dobbins RL, Szczepaniak LS, Bentley B, Esser V, Myhill J, McGarry JD. 2001. Prolonged inhibition of muscle carnitine palmitoyltransferase- 1 promotes intramyocellular lipid accumulation and insulin resistance in rats. Diabetes 50: 123-130.

24) Sterk JP, Stanley WC, Hoppel CL, Kerner J. 2003. A radiochemical pyruvate dehydrogenase assay: activity in heart. Anal Biochem 313: 179-182.

25) Pi-Sunyer FX. 1976. Resistance to ketosis during prolonged fasting by rats fed on a diet containing undecanoic acid, an odd-carbon-number fatty acid. Br J Nutr 35: 41-46.

26) Kim JY, Hickner RC, Cortright RL, Dohm GL, Houmard JA. 2000. Lipid oxidation is reduced in obese human skeletal muscle. Am J Physiol Endocrinol Metab 279: E1039- E1044.

27) Zurlo F, Lillioja S, Esposito-Del Puente A, Nyomba BL, Raz I, Saad MF, Swinburn BA, Knowler WC, Bogardus C, Ravussin E. 1990. Low ratio of fat to carbohydrate oxidation as predictor of weight gain: study of 24-h RQ. Am J Physiol 259: E650-E657.

28) Pasarica M, Tchoukalova YD, Heilbronn LK, Fang X,
Albu JB, Kelley DE, Smith SR, Ravussin E; Look AHEAD Adipose Research Group. 2009. Differential effect of weight loss on adipocyte size subfractions in patients with type 2 diabetes. Obesity (Silver Spring) 17: 19761978.

29) Fujita A, Sasaki H, Doi A, Okamoto K, Matsuno S, Furuta H, Nishi M, Nakao T, Tsuno T, Taniguchi H, Nanjo K. 2008. Ferulic acid prevents pathological and functional abnormalities of the kidney in Otsuka LongEvans Tokushima Fatty diabetic rats. Diabetes Res Clin Pract 79: 11-17.

30) Matsuda S, Iwata K, Takahashi K, Homma H, Tamura Y, Kanda Y, Inokami T, Nosaka H, Nagase M, Uchida S. 2004. A low-protein diet concomitant with high calorie intake preserves renal function and structure in diabetic OLETF rats. Clin Exp Nephrol 8: 322-330.

31) Lee Y, Naseem RH, Park BH, Garry DJ, Richardson JA, Schaffer JE, Unger RH. 2006. $\alpha$-Lipoic acid prevents lipotoxic cardiomyopathy in acyl CoA-synthase transgenic mice. Biochem Biophys Res Commun 344: 446452.

32) Zhou YT, Grayburn P, Karim A, Shimabukuro M, Higa M, Baetens D, Orci L, Unger RH. 2000. Lipotoxic heart disease in obese rats: implications for human obesity. Proc Natl Acad Sci USA 97: 1784-1789.

33) Szczepaniak LS, Dobbins RL, Metzger GJ, SartoniD'Ambrosia G, Arbique D, Vongpatanasin W, Unger R, Victor RG. 2003. Myocardial triglycerides and systolic function in humans: in vivo evaluation by localized proton spectroscopy and cardiac imaging. Magn Reson Med 49: $417-423$.

34) Le Couteur DG, Fraser R, Cogger VC, McLean AJ. 2002. Hepatic pseudocapillarisation and atherosclerosis in ageing. Lancet 359: 1612-1615.

35) Harrison SA, Brunt EM, Goodman ZD, Di Bisceglie AM. 2006. Diabetic hepatosclerosis: diabetic microangiopathy of the liver. Arch Pathol Lab Med 130: 27-32.

36) Bernuau D, Guillot R, Durand-Schneider AM, Poussier P, Moreau A, Feldmann G. 1985. Liver perisinusoidal fibrosis in $\mathrm{BB}$ rats with or without overt diabetes. Am J Pathol 120: 38-45.

37) Aumailley M, Battaglia C, Mayer U, Reinhardt D, Nischt R, Timpl R, Fox JW. 1993. Nidogen mediates the formation of ternary complexes of basement membrane components. Kidney Int 43: 7-12.

38) Kohfeldt E, Sasaki T, Göhring W, Timpl R. 1998. Nidogen-2: a new basement membrane protein with diverse binding properties. J Mol Biol 282: 99-109.

39) Bajotto G, Murakami T, Nagasaki M, Tamura T, Tamura N, Harris RA, Shimomura Y, Sato Y. 2004. Downregulation of the skeletal muscle pyruvate dehydrogenase complex in the Otsuka Long-Evans Tokushima Fatty rat both before and after the onset of diabetes mellitus. Life Sci 75: 2117-2130.

40) Curto M, Novi RF, Rabbone I, Maurino M, Piccinini M, Mioletti S, Mostert M, Bruno R, Rinaudo MT. 1997. Insulin resistance in obese subjects and newly diagnosed NIDDM patients and derangements of pyruvate dehydrogenase in their circulating lymphocytes. Int $J$ Obes Relat Metab Disord 21: 1137-1142.

41) Liu S, Manson JE. 2001. Dietary carbohydrates, physical inactivity, obesity, and the 'metabolic syndrome' as predictors of coronary heart disease. Curr Opin Lipidol 12: 395-404. 